\title{
RFI AND REMOTE SENSING OF THE EARTH FROM SPACE
}

\author{
D.M. Le Vine \\ Goddard Space Flight Center \\ Greenbelt, MD 20771
}

\author{
J. T. Johnson \\ The Ohio State University \\ Columbus, Ohio 43210 USA
}

\author{
J. Piepmeier \\ Goddard Space Flight Center \\ Greenbelt, MD 20771 USA
}

\begin{abstract}
Passive microwave remote sensing of the Earth from space provides information essential for understanding the Earth's environment and its evolution. Parameters such as soil moisture, sea surface temperature and salinity, and profiles of atmospheric temperature and humidity are measured at frequencies determined by the physics (e.g. sensitivity to changes in desired parameters) and by the availability of suitable spectrum free from interference. Interference from manmade sources (radio frequency interference) is an impediment that in many cases limits the potential for accurate measurements from space. A review is presented here of the frequencies employed in passive microwave remote sensing of the Earth from space and the associated experience with RFI.
\end{abstract}

Index Terms - Radio Frequency Interference, Remote Sensing, Passive Microwave, Radiometer

\section{INTRODUCTION}

Passive microwave remote sensing of the Earth from space provides information essential for understanding the Earth environment and its evolution. Examples include soil moisture and precipitation (important for understanding the global water cycle), ocean salinity and temperature (important for understanding ocean circulation and climate), and profiles of atmospheric temperature and humidity which are important for weather prediction and understanding global circulation. At the low end of the microwave spectrum (e.g. $1-10 \mathrm{GHz}$ ) the atmosphere is relatively transparent and measurement of surface parameters predominate. Examples are soil moisture, sea surface salinity, sea surface temperature and ocean winds. Above about $10 \mathrm{GHz}$, atmospheric attenuation becomes important and resonant lines of Oxygen $\left(\mathrm{O}_{2}\right)$ and water $\left(\mathrm{H}_{2} \mathrm{O}\right)$ appear which can be used to obtain profiles of atmospheric temperature and water vapor, and to monitor precipitation and clouds. The troughs between peaks of attenuation associated with the resonances can be used to observe the surface and produce parameters such as the extent and age of sea ice.
The frequencies employed in passive microwave remote sensing of the Earth from space are determined by the sensitivity of natural (i.e. thermal) emission to the parameter of interest and by the availability of suitable spectrum [24]. The latter is especially a problem at the lower end of the microwave spectrum, which is crowded and increasingly in demand for growing applications such as phone and internet communication and TV broadcast. It takes little leakage from these systems and from strong sources such as air traffic control radar to completely mask the weak natural "thermal" emission from the Earth surface and atmosphere upon which passive sensors in space depend.

\section{EXAMPLE: REMOTE SENSING OF SALINITY}

Remote sensing of sea surface salinity will be used as an example to illustrate how the choice of frequency is made. Surface salinity reflects the balance between evaporation and precipitation and is helpful for understanding the global water cycle and important for understanding ocean circulation. Salinity affects the conductivity of seawater which in turn affects the thermal emission from the water. Although the signal is small, the changes in the natural emission from the ocean surface associated with changes in salinity can be measured from space. This is illustrated in Fig 1 which shows the sensitivity, dTB/dS, of the emission

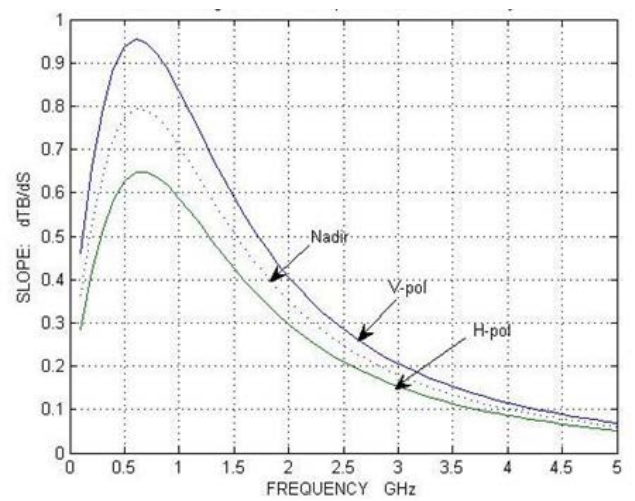

Fig 1: The sensitivity of changes in brightness temperature to changes in salinity for SST $=20 \mathrm{C}$ and $\mathrm{S}=35$ PSU. The solid curves are for an incidence angle of 40 degrees. 
(brightness temperature, TB) to changes in salinity (S) as a function of frequency [10]. Theses curves were derived using the Klein-Swift model function [9] for the dielectric constant of seawater with temperature $=20 \mathrm{C}$ and S $=30 \mathrm{psu}$ and a flat surface. The solid curves are for an incidence angle of 40 degrees and the dashed curve is for observations at nadir. Typical requirements for surface salinity for applications in the open ocean are on the order of 0.1 psu which requires an accurate, stable radiometer with a sensitivity on the order of $0.1 \mathrm{~K}$. This is challenging, and requires bandwidth and integration time to reduce noise. The obvious choice would be to operate at the peak of the curve (about $600 \mathrm{MHz}$ ), but this part of the spectrum is a very crowded and one must also consider spatial resolution (low frequency means large antennas). Fortunately, there is a window of $27 \mathrm{MHz}$ centered at $1.413 \mathrm{GHz}$ which has been preserved for passive use only, which is not far from the peak in sensitivity, and is on the high frequency side to reduce antenna aperture requirements. With this bandwidth plus spatial averaging and good radiometer design, it is possible to measure surface salinity. The European Space Agency mission SMOS and NASA's Aquarius mission have demonstrated the feasibility of this measurement $[11,12,19]$. Were it not for this protected portion of the spectrum, this measurement would not have been possible.

\section{FREQUENCIES USED IN REMOTE SENSING FROM SPACE}

Table 1 lists thel frequencies commonly employed and some of the science applications made with the measurements. For example, ocean salinity, and also soil moisture, are made using the protected window at $1.4 \mathrm{GHz}$. Most other applications involve more than one frequency. Generally, these consist of a primary frequency, where the sensitivity to the primary geophysical parameter of interest is strong, and other frequencies which help correct for competing factors such as atmospheric attenuation. The measurement of sea surface temperature (SST) is an example. The sensitivity to changes in SST peaks in the vicinity of $6 \mathrm{GHz}$. Surface roughness (waves) also contributes to the brightness temperature and attenuation due the atmosphere can be important. Existing algorithms to retrieve SST (e.g. [22]) use a primary frequency of $6.9 \mathrm{GHz}$ combined with other frequencies $(10.7,18.7,23.8$, and $36.5 \mathrm{GHz}$ ) to address these competing factors. The 10.7 and $18.7 \mathrm{GHz}$ measurements help correct for roughness and 23.8 and 36.5 $\mathrm{GHz}$ help correct for the effects of water in the atmosphere.

Table II is an historical list of passive microwave sensors and the frequencies employed. It is not a complete list (it has a NASA focus) but illustrates that the choice dates back to the earliest days of passive microwave remote sensing of the Earth from space. The consistency of the choice of frequency should not be surprising since the applications (and associated physics) and spectrum allocations have not changed. The table also includes several new missions in the planning stages such as a cubesat mission designed for research on RFI called CubeRRT [7]. In this table "wv" stands for the water vapor resonance at $23 \mathrm{GHz}$ and " $\mathrm{G}$ " represents frequencies above $100 \mathrm{GHz}$ including the water vapor resonance at $183 \mathrm{GHz}$ and several above that used primarily for limb sounding of atmospheric chemistry.

Table III presents a more detailed look at the frequencies employed for several recent and future passive microwave sensors in space. The top three rows list (respectively) the nominal frequency, indicate if it is protected for passive use only (green) or shared with other services (red), and give the center frequency and bandwidth of those bands that are protected. The remainder of the table lists the frequencies and bandwidth employed by each sensor. For example, SMOS, Aquarius and SMAP are single frequency radiometers operating in the protected band at $1.41 \mathrm{GHz}$ (with $27 \mathrm{MHz}$ of bandwidth centered at $1.413 \mathrm{GHz}$ ).

Table I: Frequencies commonly use in passive microwave remote sensing. Solid circles indicate primary measurements.

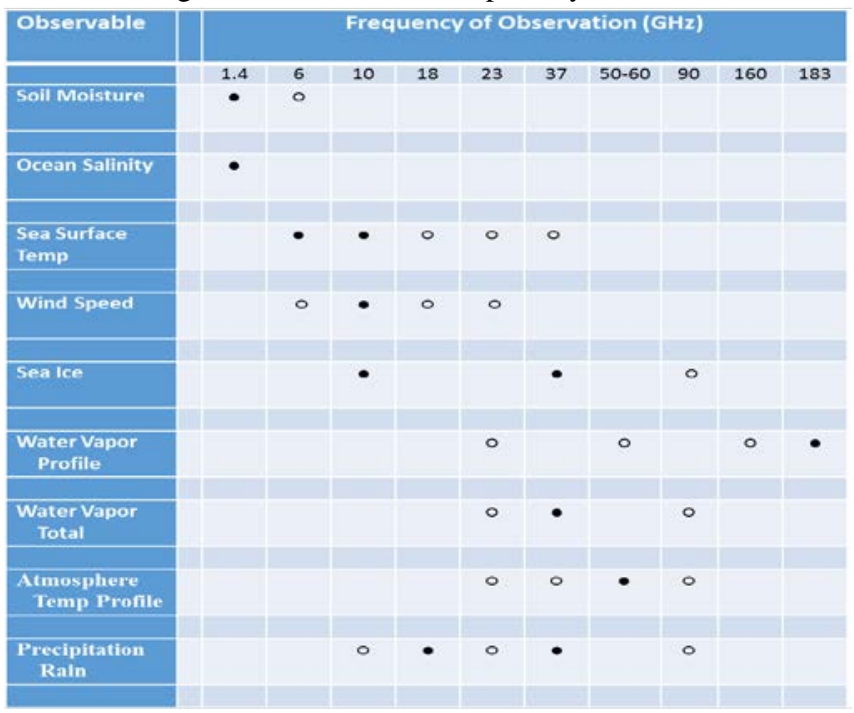

Table II: Historical perspective on frequency band employed in NASA passive microwave remote sensing of the Earth from space.

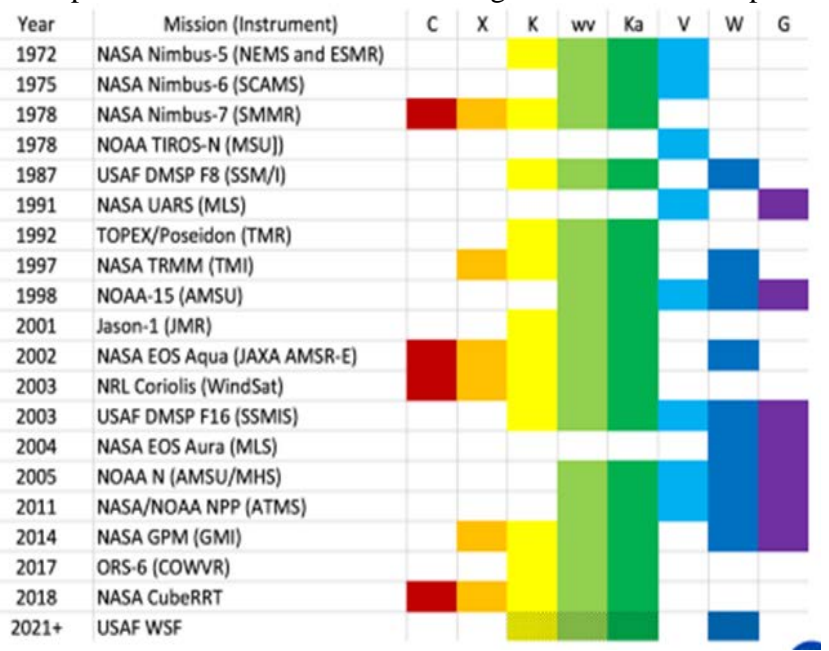


Table III. Frequency and bandwidth used by several example sensors in space. Notes: *these bands are protected; $\wedge$ Shared bands with some protection; \# 50.2 -50.4 GHz and 52.60-54.25 GHz.

\begin{tabular}{|c|c|c|c|c|c|c|c|c|c|c|}
\hline \multirow[t]{2}{*}{ Sensor } & \multicolumn{5}{|c|}{ Frequency in $\mathrm{GHz}$} & \multicolumn{3}{|c|}{ (Bandwidth) in $\mathrm{MHz}$} & \multirow[b]{2}{*}{165} & \multirow[b]{2}{*}{183} \\
\hline & 1.4 & 6.5 & 10.7 & 18.7 & 23 & 37 & 50.60 & 89 & & \\
\hline Protection & Yes. & Non & Yes & Nor & Yes & Non & Yes & Yes & yes & Yes \\
\hline $\begin{array}{l}\text { Center Freq } \\
\text { Bandwidth }\end{array}$ & $\begin{array}{l}1.413 \\
(27)\end{array}$ & & $\begin{array}{c}10.69 \\
(20)\end{array}$ & $\begin{array}{l}18.70 \\
(200)\end{array}$ & $\begin{array}{l}23.80 \\
(400)\end{array}$ & & $\#$ & $\begin{array}{c}89.0 \\
(6000)\end{array}$ & & $\begin{array}{r}183.5 \\
(2500)\end{array}$ \\
\hline \multicolumn{11}{|l|}{ Sensor } \\
\hline $\begin{array}{l}\text { SMOS/SMAP } \\
\text { Aquarius }\end{array}$ & $\begin{array}{l}1.4 \\
\text { (27) }\end{array}$ & & & & & & & & & \\
\hline AMSR-2 & & $\begin{array}{l}6.9 / 7.3 \\
(350)\end{array}$ & $\begin{array}{l}10.65 \\
(100)\end{array}$ & $\begin{array}{l}18.7 \\
(200)\end{array}$ & $\begin{array}{l}23.8 \\
(400)\end{array}$ & $\begin{array}{c}36.5 \\
(1000)\end{array}$ & & $\begin{array}{c}89.0 \\
(3000)\end{array}$ & & \\
\hline GMI & & & $\begin{array}{l}10.65 \\
(100)\end{array}$ & $\begin{array}{l}18.7 \\
(200)\end{array}$ & $\begin{array}{l}23.8 \\
(370)\end{array}$ & $\begin{array}{l}36.64 \\
(700)\end{array}$ & & $\begin{array}{l}89.0 \\
(2750)\end{array}$ & $\begin{array}{l}165.6 \\
(1570)\end{array}$ & $\begin{array}{l}18312 \\
(1480)\end{array}$ \\
\hline ATMS & & & & & $\begin{array}{l}23.8 \\
(270)\end{array}$ & $\begin{array}{l}31.4^{*} \\
(180)\end{array}$ & $\begin{array}{l}\text { SS(11) } \\
(400)\end{array}$ & $\begin{array}{c}88.2 \\
(2000)\end{array}$ & $\begin{array}{l}165.5 \\
(2000)\end{array}$ & $\begin{array}{l}183(5) \\
(2000)\end{array}$ \\
\hline SSMIS & & & & $\begin{array}{l}19.35 \\
(360)\end{array}$ & $\begin{array}{r}22.24^{*} \\
(400)\end{array}$ & $\begin{array}{c}37.0 \\
(1600)\end{array}$ & $\begin{array}{l}50-63 \\
(300)\end{array}$ & $\begin{array}{l}91.66 \\
(350)\end{array}$ & $\begin{array}{l}150^{*} \\
(1650)\end{array}$ & $\begin{array}{l}183(3) \\
(1500)\end{array}$ \\
\hline
\end{tabular}

Several sensors operate at C-band (6-7 GHz) including AMSR-2 and its predecessors. This band is not protected for passive use. It is shared with communication services (such as fixed point-to-point communication channel relay links) and problems with RFI over land are an issue. Also, notice the yellow color for the band at $10.7 \mathrm{GHz}$. There is a protected window at X-band $(10.7 \mathrm{GHz})$, but it is relatively narrow (20 MHz) and sensors using this band typically employ larger bandwidth.

\section{EXPERIENCE WITH RFI}

Radio frequency interference (RFI) has a long history in passive microwave remote sensing from space, especially at the low frequency end of the spectrum [14]. Even operating in a protected band is no guarantee that the observations will be free of RFI, as the examples below will illustrated.

\subsection{RFI at L-Band}

This is illustrated in recent experience with SMOS, Aquarius and SMAP, each of which have experienced significant RFI, especially over land [11, 15, 17] even though they are single frequency radiometers which measure in the protected band at $1.413 \mathrm{GHz}$. Figure 2 is an example of the RFI experienced by Aquarius [11]. This is a plot of the percentage of data flagged as RFI averaged over one year. In areas colored red, almost all the data is lost to RFI.

The sources of strong RFI show a strong correlation with centers of population. This is particularly evident in the Americas. Also, apparent is the line of radar systems along the top of North America that comprise the Distant Early Warning system (DEW line). The problem over land was even worse when SMOS launched in 2009, but has gradually improved over Europe as the result of ESA efforts to report RFI and work with national spectrum management authorities of enforce ITU regulations [17].

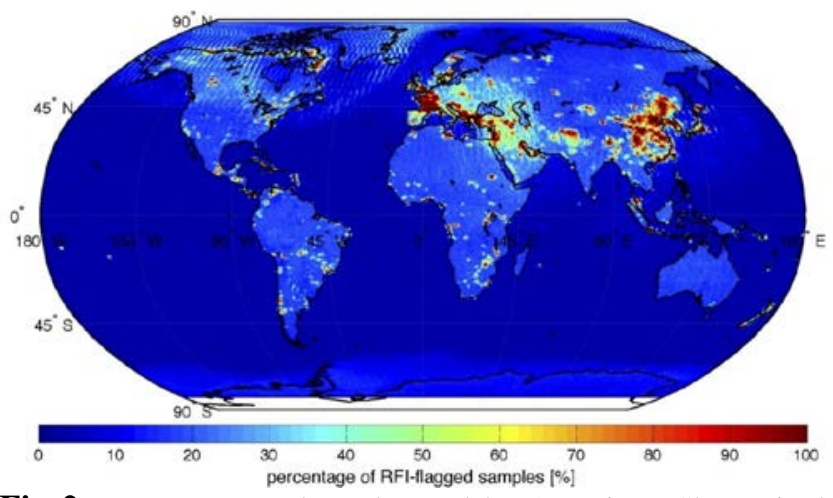

Fig 2. RFI at L-Band as observed by Aquarius. Shown is the percentage of samples flagged as RFI averaged for one year (Aug 2011- Aug 2012). Both polarizations are included in the average.

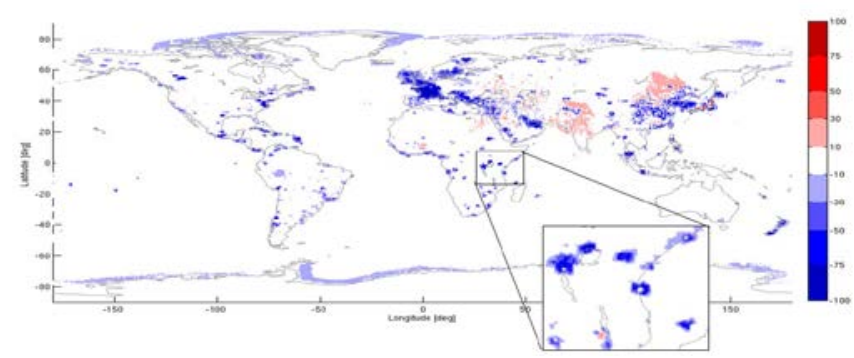

Fig 3. Global difference between SMOS and Aquarius RFI: SMOS RFI percentage - Aquarius RFI percentage.

The sources are real, but RFI also appears where there are no sources in the main beam of the antenna. This is evident in the halo of RFI in Fig 2 in the North Atlantic running from east of Greenland south toward the east coast of the USA. This is RFI due to strong sources in North America and entering via the antenna sidelobes. This is confirmed by comparing ascending and descending passes and different polarizations [11]. This example points out an important issue with RFI: although the sources are real and distinct, the way RFI appears at the sensor depends on the characteristics of the sensor (e.g. also see [1]).

Figure 3 shows the difference in RFI reported by Aquarius and SMOS. Both radiometers operate in the protected band at $1.4 \mathrm{GHz}$ but in other ways are very different. Aquarius is a conventional system consisting of three independent radiometers operating in pushbroom fashion whereas SMOS is an interferometer that forms images through software using the correlation of signal from pairs of elements in its array [8]. The RFI algorithms are also much different: Aquarius over samples and filters based on statistical fluctuations from the mean which is adjusted in real time [11], whereas SMOS uses a fixed threshold for detection [20]. In total, Aquarius tends to be more sensitive than SMOS, while SMOS has better spatial resolution. This is evident in Figure 3 which shows the difference in RFI detected by each system. On a global scale, the distribution of sources are similar, but with different size and at different levels. This is illustrated in the insert over Africa shown 
with finer resolution. With better resolution the differences appear to have a white center (no difference; probably because each sensor is saturated) but with a blue halo indicating that Aquarius sees a larger region of RFI around the source. However, there are exceptions such as the red areas in China and India indicating that SMOS sees more RFI than Aquarius. This also appears to be the case in Japan [21]. Recent research into the nature the RFI in Japan suggests that this is due, at least part, to emission from consumer satellite TV receivers. The RFI is detected by SMOS because the signal level is raised, but missed by Aquarius because the net appears random and just raises the local mean. Similar examples have been found in Russia and a threshold is being added to the Aquarius detection algorithm to address such sources.

\subsection{RFI at C-Band}

Over the ocean, C-band (6-7 GHz) is important for monitoring sea surface temperature (SST) because it is close to the peak sensitivity of the radiometer output to changes in water temperature. Attempts to use this frequency over land, for example to retrieve soil moisture, have encountered significant RFI. This is a shared band (communications services have primary allocations), and aircraft experiments have indicated the presence of RFI [5, 6]. This was confirmed when C-band data from AMSR-E became available (e.g.[16]) which showed significant RFI over the USA and the Middle East detected using the mean and standard deviation of "spectral indices", the difference between the brightness temperature at two adjacent frequencies, C-band $(6.9 \mathrm{GHz})$ and X-band $(10.7 \mathrm{GHz})$ in this case. Fig 4 showing the spectral indices derived from WindSat data [3] is another example of significant RFI at Cband over the USA. Fig 4 is at vertical polarization and the data in horizontal polarization is similar. Other indicators, such as simple extremes of C-band brightness temperature have a similar appearance.

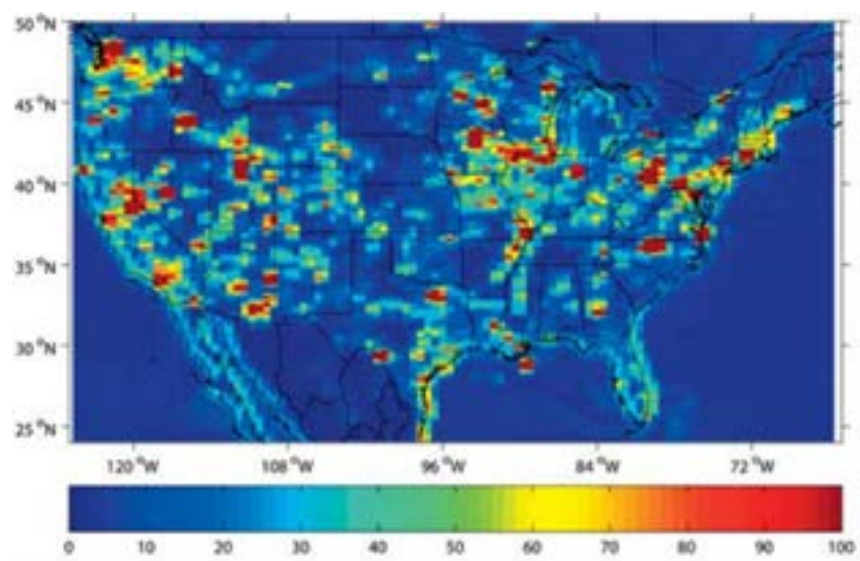

Fig 4. Maximum value of the spectral index (difference between C- and X-band) at V-pol. From Ellingson and Johnson [3].

Global studies of RFI at C-band show that the use of this band varies significant by country, with larger interference in the Middle East, Asia, and Japan, and lesser use in other regions. While larger sources of RFI are easily identified, lower level RFI is often indistinguishable from geophysical contributions, resulting in erroneous science products. The RFI in this band has significantly impeded efforts to produce soil moisture and other land surface products using $6.9 \mathrm{GHz}$.

To address the RFI problem at C-band AMSR-2 used two channels. Differences in brightness temperatures between the two channels are used to indicate the presence of RFI and the lower amplitude channel is selected in for use in retrievals [23].

\subsection{RFI at $\mathrm{X}-\mathrm{Band}$}

The channel at $10.7 \mathrm{GHz}$ (X-band) is important for monitoring sea surface temperature and also winds over the ocean. The $20 \mathrm{MHz}$ band between 10.68 and $10.70 \mathrm{GHz}$ is protected for passive use only. However, current radiometers use a wider bandwidth of $100 \mathrm{MHz}$ at this frequency (e.g. Table III). RFI over land at this frequency has been reported over various portion of the globe [16] and the USA [3]. The frequency allocations for bands surrounding this protected spectrum include space-based TV broadcast satellite services, and interference from such services has also been reported over the ocean. In particular, [4] reports RFI in AMSR-E data over the ocean which is directly attributable to geo-stationary broadcast satellites. This was confirmed by ray tracing using the ray from the satellite reflected from the surface back to the source. In particular, Fig. 3 of [4] presents a history of this interference due to reflection from the ocean from several locations for a period of 9 years. The RFI has a mean value which trends upward indicating an increasing amount of interference. The presence of RFI was inferred by comparing results of the SST retrieval algorithm with and without the X-band (10.7 GHz) data. The difference was examined for outliers, and the outliers flagged as RFI.

\subsection{RFI at K-Band (18.7 GHz)}

RFI over the continental USA has been reported at K-band (18.7) GHz from Windsat and AMSR-E [13]. For example, [13] reports RFI at $18.7 \mathrm{GHz}$ from WindSat derived using the difference between observations at 23.8 and $18.7 \mathrm{GHz}$ (the assumption being that large differences between close frequencies are due to non-geophysical sources.) The data indicate an increase in RFI from 2005 to 2008 and a seasonal dependence. Evidence also exists that RFI is due to signal from satellite downlinks reflected from the surface.

\section{5. RFI at K-Band (20-24 GHz)}

Although evidence of RFI in the $20-24 \mathrm{GHz}$ band has been reported less frequently, interference remains a subject of concern, in particular given the growing penetration of wide bandwidth automotive radar systems operating at these 
frequencies. This band is of crucial importance for atmospheric water content monitoring and the correction of atmospheric attenuation in surface observations with other frequencies. Figure 5 illustrates maximum brightness temperatures encountered through March 2014 in the 21.3 $\mathrm{GHz}$ vertically polarized channel of the Tropical Rainfall Measuring Mission's (TRMM) Microwave Imager (TMI), which observes at nadir. Maximum brightness temperatures are useful only for locating extreme RFI values, and cannot illustrate lower levels of RFI corruption. Nevertheless, the results show evidence of RFI corruption, particularly in Japan. The plot on the right shows cross sections of the image in latitude through Japan, and includes all of TMI's frequencies. The high values (sometimes greater than 400 $\mathrm{K}$ ) clearly show that RFI is present in the $21.3 \mathrm{GHz}$ band.

\subsection{RFI at Higher Frequencies}

RFI at frequencies of above $23 \mathrm{GHz}$ has been mentioned, but attenuation by the atmosphere helps protect observations from distant sources. However, new applications continue to be proposed with the potential to threaten remote sensing (e.g. the development of WiFi for aircraft passengers at frequencies near the water vapor resonance at 50-60 GHz).

\section{TRENDS IN RFI DETECTION AND MITIGATION}

Other than the L-band examples presented in Section 4.1, RFI detection has largely been after the fact. That is, except for a threshold at the radiometer to identify extreme values, the presence of RFI has been inferred by looking for anomalies in the processed brightness temperatures or science products. The L-band missions represent a different approach in which specific plans were made prior to launch to detect RFI at the radiometer. Prior to launch, aircraft experiments (e.g. [18]) indicated RFI was going to be a problem, and anticipating the issue, design changes were made specifically to detect RFI. For example, in the case of Aquarius, the radiometer was designed to sample at a very high rate (one sample each $10 \mathrm{~ms}$ ) and an algorithm was developed to use these samples to develop statistics which could be used to identify RFI [11]. The samples flagged as RFI are removed, and the remaining data averaged and then sent for processing (calibration and retrieval of salinity). Oversampling in time allows statistics to be developed and
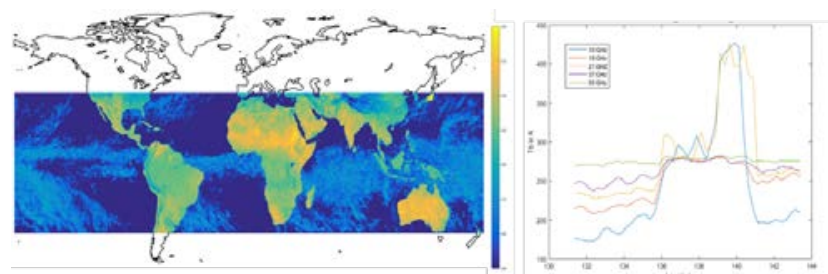

Fig 5: Maximum brightness temperatures observed by the Tropical Rainfall Measuring Mission's (TRMM) Microwave Imager (TMI) in March 2014, vertical polarization, 21.3 GHz. Left plot illustrates the maximum brightness temperature reported, while the right plot illustrates values along longitude 139.375 (through Japan.) samples to be removed without completely losing a given footprint measurement to RFI.

SMAP takes the process one step further. The SMAP receiver also oversamples in time, but also divides the 24 $\mathrm{MHz}$ L-band received signal into 16 subbands. SMAP then applies RFI detection algorithms developed to detect pulsed interference, narrowband interference, and other sources of interference using the kurtosis statistic [15]. Fig 6 is an example illustrating SMAP's RFI processing aspects. The data was obtained from a portion of a scan overpassing Japan. The lower portions of the figure show SMAP's 16 subchannel spectrogram (in this case for multiple footprints) before (left) and after (right) RFI detection algorithms are applied to remove corrupted portions of the spectrogram. In this case, extreme RFI corruption results in loss of footprint measurements during this overpass [15]. While SMAP's RFI processor is capable of detecting and removing some RFI corrupted portions of the spectrogram, doing so reduces the measurement sensitivity and results in a degraded measurement of the desired geophysical products.

Research is underway into the possibility of extending RFI processing to other frequencies and in channels with larger bandwidths than the $24 \mathrm{MHz}$ used at L-band. In particular, a cubesat experiment, CubeRRT [6] is being prepared for launch in 2018 which will collect data over a wide frequency range $(6-40 \mathrm{GHz})$ using an RFI processing backend similar to that flown on SMAP but having a $1 \mathrm{GHz}$ instantaneous bandwidth. Unlike SMAP, which implemented RFI detection and removal in ground processing, CubeRRT will perform RFI detection and removal onboard the spacecraft. Onboard processing is desirable with larger channel bandwidths to avoid the
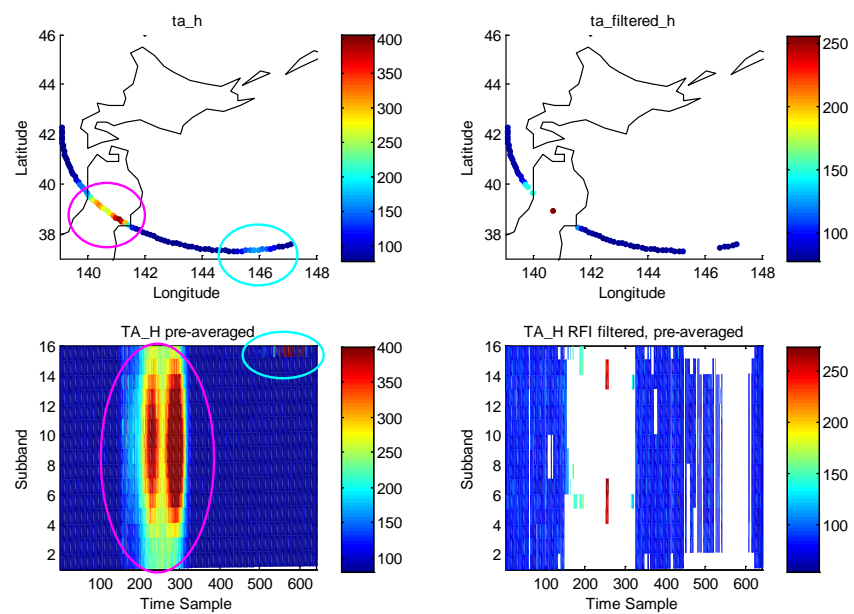

Fig 8: Plot of the first 80 footprints of horizontally polarized SMAP antenna temperatures within a scan over Honshu, Japan taken on June 3, 2015. The top left are geolocated footprints before RFI mitigation; the top right are geolocated footprints after RFI mitigation; the bottom left are corresponding frequency-time data before RFI filtering and footprint averaging; the bottom right show the same data as the bottom left plot after RFI filtering. 
requirement for downlinking large volumes of data, particularly given the larger channel bandwidths typically desired at higher radiometric frequencies.

\section{CONCLUSION}

RFI is a problem for passive microwave remote sensing of the Earth from space. The problem likely will get worse. New applications, more sensitive radiometers and the need for additional bandwidth will make protection from RFI more important. On the other side, commercial pressure for more spectrum for communication and other services will continue to put pressure on an already crowded spectrum. $\mathrm{R}$ research is needed to find new ways for the competing needs of science and commercial applications to co-exist.

\section{REFERENCES}

[1]. M. Aksoy and J. T. Johnson, “A Comparative Analysis of Low-Level Radio Frequency Interference in SMOS and Aquarius Microwave Radiometer Measurements,” IEEE Trans. Geosc. Rem. Sens., vol. 51, pp. 4983-4992, 2013.

[2]. S. K. Chan et al, "Assessment of the SMAP Passive Soil Moisture Product,” IEEE Trans. Geosc. Rem. Sens., vol. 54, pp. 4994-5007, 2016.

[3]. S.W. Ellingson and J.T. Johnson, “A Polarimetric Survey of Radio-Frequency Interference in $\mathrm{C}$ - and $\mathrm{X}$-Bands in the Continental United States Using WindSat Radiometry”, IEEE Trans. Geosci. Remote Sens., vol. 44 (3), pp. 540-548, 2006.

[4]. K. Hilburn, C. Gentemann, M. Brewer, L. Ricciardulli, and F. Wentz, "RFI Detection and Mitigation for AMSR-E Ocean Retrievals”, Proc. MicroRad 2014.

[5]. Jackson, et al., "Soil Moisture Retrieval Using the C-Band Polarimetric Scanning Radiometer During the Southern Great Plains 1999 Experiment,” IEEE Trans. Geosci. Remote Sens , Vol 40 (no. 10), October, 2002.

[6]. J. T. Johnson et al, "Airborne radio frequency interference studies at C-band using a digital receiver," IEEE Trans. Geosc. Rem. Sens., vol. 44, pp. 1974-1985, 2006.

[7]. J. Johnson et al, "CubeRRT: The CubeSAT Radiometer Radio Frequency Interference Technology Validation Mission," IGARSS 2016, conference proceedings, 2016.

[8]. Y. Kerr et al, "Soil Moisture Retrieval From Space: The Soil Moisture and Ocean Salinity (SMOS) Mission,” IEEE Trans. Geosc. Rem. Sens., vol. 39, pp. 1729-1735, 2002

[9]. L.A. Klein and C.T. Swift, "An improved model for the dielectric constant of sea water at microwave frequencies", IEEE Trans. Ant \& Propag., Vol. AP-25 (1), pp.104, 1977.

[10]. D.M. Le Vine, G.S.E Lagerloef and S.E. Torrusio, “Aquarius and Remote Sensing of Salinity from Space”, Proc. IEEE,Vol 98 (\#5), pp 688-703, May, 2010.
[11]. D. M. Le Vine, P. de Matthaeis, C. S. Ruf, and D. D. Chen, "Aquarius RFI detection and mitigation algorithm: Assessment and Examples,” IEEE Trans. Geosci. Remote Sens., vol. 52, no. 8, pp. 4574-4584, 2014.

[12]. D.M. Le Vine et al, "Status of Aquarius/SAC-D and Aquarius Salinity Retrievals”, IEEE J. of Selected Topics in Applied Earth Observation and Remote Sensing (JSTARS), Vol 8 (\#12), pp 5401-5415, December, 2015.

[13]. D. McKague, J. Puckett, and C. Ruf, "Characterization of Kband radio frequency interference from AMSR-E, WindSat and SSM/I," Proc. Int. Geoscience Remote Sensing Symp., Honolulu, HI, July 25, 2010, pp. 2492-2494.

[14]. S. Misra and P. de Matthaeis, "Passive Remote Sensing and Radio Frequency Interference (RFI)”, IEEE GRSS Magazine, PP 68-73, June 2014.

[15]. P. Mohammed et al, "SMAP L-Band Microwave Radiometer: RFI Mitigation Prelaunch Analysis and First Year On-Orbit Observations,” IEEE Trans. Geosc. Rem. Sens., vol. 54, pp. 6035-6047, 2016.

[16]. E. Njoku, P. Ashcroft, T.K. Chan and Li Li, "Global Survey and Statistics of Radio-Frequency Interference in AMSR-E Land Observations”, IEEE Trans. Geosci. Remote Sens., vol. 43, no. 5, pp. 938-947, May, 2005.

[17]. R. Oliva et al, "SMOS Radio Frequency Interference Scenario: Status and Actions Taken to Improve the RFI Environment in the 1400-1427-MHz Passive Band,” IEEE Trans. Geosc. Rem. Sens., vol. 50, pp. 1427-1439, 2012.

[18].J. Park et al, “Airborne L-Band Radio Frequency Interference Observations From the SMAPVEX08 Campaign and Associated Flights,” IEEE Trans. Geosc. Rem. Sens., vol. 49, pp. 3539-3570, 2011.

[19]. N. Reul, et al, "Sea Surface Salinity Observations from Space with the SMOS Satellite: A New Means to Monitor the Marine Branch of the Water Cycle,” Surveys in Geophysics, vol. 35, pp. 681-672, 2014.

[20]. Y. Soldo, D.M. Le Vine, P. de Matthaeis, P. Richaume, "LBand RFI Detected by SMOS and Aquarius, ”, IEEE Trans. Geosci. Remote Sens., submitted Septemer, 2016.

[21]. Y. Soldo, P. De Matthaeis and D.M. Le Vine, "L-Band RFI in Japan”, Proc. RFI 2016 Conference, Socorro, NM, Oct, 2016.

[22]. F. Wentz et al, "Satellite Measurements of Sea Surface Temperature through Clouds,” Science, vol. 288, pp. 847-850, 2000 .

[23]. E. Zabolotskikh et al, "Radio-Frequency Interference Identification Over Oceans for C- and X-Band AMSR2 Channels," Geosc. Rem. Sens. Let., vol. 12, pp. 1705-1709, 2015.

[24]. J. Judage et al, "Handbook of Frequency Allocations and Spectrum Protection for Scientific Uses, $2^{\text {nd }}$ Ed., National Academies Press, Washington, DC, 2015. 